\section{Comparison of Irbesartan and Benazepril Hydrochloride on Urine Protein Reduction in Chronic Glomerulonephritis}

\section{Sir,}

Chronic glomerulonephritis (CGN) is a primary glomerular disease, with the main clinical manifestations of proteinuria, hematuresis, edema, and hypertension. CGN is characterised by insidious onset and delayed course of disease, and most patients may progress to end-stage renal disease. ${ }^{1}$ Proteinuria is not just an important clinical mark of glomerular basement membrane damage of CGN, but also an independent risk factor which makes the kidney further damaged. Controlling proteinuria has become a main target of CGN treatment. Angiotensin II receptor blocker (ARB) agent irbesartan and angiotensin converting enzyme inhibitor (ACEI) agent benazepril hydrochloride have therapeutical effect on hypertension. ${ }^{2,3}$ But, there are few studies about the comparison of effects of irbesartan and benazepril hydrochloride on urine protein reduction in patients with CGN.

The objective of this study was to compare the effect of irbesartan and benazepril on the proteinuria in patients with CGN.

A total of 90 patients with CGN, received and managed in Changyi City People's Hospital, China, fromJanuary 2018 to May 2019, were selected as the research subjects. This study was approved by the Hospital Ethics Committee. An informed consent was signed by all patients. Inclusion criteria were patients with conformity to clinical diagnosis criteria of CGN, clinical manifestation urinary total protein (UTP, $0.5 \mathrm{~g} / 24$ hours $\leq$ UTP $<3.5 \mathrm{~g} / 24$ hours), initial onset, without use of ARB and ACEI medicines, and aged $18-70$ years old. Exclusion criteria were patients with diabetes, primary hypertension and other secondary glomerular diseases, UTP $\geq 3.5 \mathrm{~g} / 24$ hours, combined with tumor disease, pregnant or lactating women, aged below 18 years and above 70 years, abnormal liver function, clinical observation time was less than 8 weeks, the factors leading to microalbuminuria such as aggravating activity, fever, infection and cardiac insufficiency. According to a random number table method, the patients were randomly divided into irbesartan group and benazepril hydrochloride group, with 45 cases in each group.

Conventional basic therapy was given for each group, including anti-infection, lipid-lowering and anticoagulant therapy as well as correction of acidosis and electrolyte imbalance. Irbesartan was given for irbesartan group by oral administration, $150 \mathrm{mg}$ per time $\square$ one time a day and continuously for eight weeks. Benazepril hydrochloride was used for benazepril hydrochloride group by oral administration, $10 \mathrm{mg}$ per time, one time a day and continuously for eight weeks. The main outcome variable, i.e. 24hour urine protein, was determined by radioimmunoassay in patients of both groups before treatment and after treatment. At the end of the 8-week of treatment, the curative effect of urinary protein was judged by clinical control, markedly effective, effective and invalid. Curative effect of urinary protein was judged as clinical control, if the routine urine test results were negative, or the 24-hour urine protein excretion was normal. Curative effect of urinary protein was judged as markedly effective, if the routine urine test results decreased by two "+", or the 24-hour urine protein excretion decreased by more than $40 \%$; as effective if the routine urine test results decreased by one "+", or 24-hour urine protein excretion decreased by less than $40 \%$; and as invalid if there was no decrease, or even an increase in urinary protein excretion. The total treatment efficiency rate $(\%)=$ (clinical control cases + markedly effective cases + effective cases) / total number of cases $\times 100 \%$. Adverse reactions were recorded. Data were analysed by SPSS version 25.0. Frequencies and percentages were calculated for categorical variables, examined by Chi-square test. Mean \pm SD was calculated for numerical variables, examined by independent sample t-test; $p$ $<0.05$ was considered statistically significant.

Among the 90 patients, there were 53 (58.89\%) males and 37 (41.11\%) females. They were aged between 20 and 68 years, with the average of $49.64 \pm 3.75$ years. Before treatment, urine protein excretion of irbesartan group was $1.95 \pm 0.33(\mathrm{~g} / 24$ hours), while it was $2.02 \pm 0.49$ (g/24 hours) for benazepril hydrochloride group, the difference of both groups was not statistically significant $(p=0.442)$. Eight weeks after treatment, urine protein excretion of irbesartan group was $1.43 \pm 0.31$ (g/24 hours), higher than that of benazepril hydrochloride group $1.29 \pm 0.24$ ( $\mathrm{g} / 24$ hours), the difference of both groups was statistically significant $(p=0.019)$. At the end of the 8-week of treatment, the total treatmentefficiency rate of benazepril hydrochloride group was $88.89 \%$ (40 cases), higher than that of irbesartan group $71.11 \%$ (32 cases) ( $p=0.035$, Table I). During the course of 8 -week treatment, there was one headache case $(2.22 \%)$ in each group, but the difference was not statistically significant in the two groups $(p=1.000)$. The otheradverseeffects such as dizziness, drowsiness, tiredness, cough, muscle weakness, tachycardia symptoms, low blood pressure (SBP $<90 \mathrm{mmHg}$ ), hyperkalemia, abnormal liver function were not observed.

The result of this study showed that, based on conventional treatment of chronic glomerulo nephritis, ARB drug irbesartan and ACEI drug benazepril hydrochloride could reach the purpose of urine protein reduction; but the latter was superior to the former in terms of urine protein reduction. One study indicated that benazepril hydrochloride might improve diabetic nephropathy and reduce proteinuria through lowering angiopoietin-like protein-4 expression. ${ }^{4}$ Although this study results prompted that ACEI drug benazepril hydrochloride could reduce urine protein excretion of CGN patients, the risk of hyperkalemia should be noticed in clinical application. 
Table I: Comparison of curative effect of urinary protein in the two groups [n (\%)].

\begin{tabular}{|c|c|c|c|c|c|c|c|}
\hline Groups & $\mathbf{n}$ & Clinical control & $\begin{array}{l}\text { Markedly } \\
\text { effective }\end{array}$ & Effective & Invalid & $\begin{array}{c}\text { Total treatment } \\
\text { efficiency rate }\end{array}$ & p-value \\
\hline Irbesartan group & 45 & $8(17.78)$ & $6(13.33)$ & $18(40.00)$ & $13(28.89)$ & $32(71.11)$ & \multirow{2}{*}{0.035} \\
\hline Benazepril hydrochloride group & 45 & $12(26.67)$ & $8(17.78)$ & $20(44.44)$ & $5(11.11)$ & $40(88.89)$ & \\
\hline
\end{tabular}

If obvious tiredness, muscle weakness, cold hands and feet, slow reaction or drowsiness appears in the medication period, these may be caused by hyperkalemia, so it is required to immediately stop the use of ACEI agent benazepril hydrochloride. This is because ACEI agent can inhibit aldosterone secretion, thus leading to the rise of serum potassium. ${ }^{5}$ Thus, it is required to detect serum potassium concentration of benazepril hydrochloride.

Due to the small number of cases and the short time of clinical observation in this study, the exact effects of benazepril hydrochloride on reducing urine protein and delaying nephropathy progress still remain large-scale clinical tests for verification.

\section{CONFLICT OF INTEREST:}

Authors declared no conflict of interest.

\section{AUTHORS' CONTRIBUTION:}

ZL: Responsible for the conception or design of the work; drafting the work.

LZ: Responsible for acquisition, analysis, and interpretation of data for the work.

RZ: Responsible for the acquisition of data for the work; drafting the work.

JL: Revised it critically for important intellectual content; final approval of the version to be published.

\section{REFERENCES}

1. Novak SM, Caraveo JM, Trowbridge AA, Peterson RF, White RR. Progress in the treatment of chronic glomerulonephritis with traditional Chinese medicine. Cheminform 2005; 36:3542-52.

2. Han SH, Oh GC, Kwon HM, Park CG, Kim IJ, Hwang GS, et al.
Comparison of efficacy and safety between two different irbesartan generic vs. branded in the treatment of Korean patients with mild-to-moderate hypertension: An 8-week, multicenter, randomized, open-label, Phase IV clinical study. Drug Des Devel Ther 2018; 12:4217-29.

3. Jiang S, Pan M, Wu S, Venners SA, Zhong G, Hsu YH, et al. Elevation in total homocysteine levels in Chinese patients with essential hypertension treated with antihypertensive benazepril. Clin Appl Thromb Hemost 2016; 22(2):191-8.

4. Xue L, Feng X, Wang C, Zhang X, Yu K. Benazepril hydrochloride improves diabetic nephropathy and decreases proteinuria by decreasing ANGPTL-4 expression. BMC Nephrology 2017; 18(1):307.

5. Puig JG, Marre M, Kokot F, Fernandez M, Jermendy G, Opie L, et al. Efficacy of indapamide SR compared with enalapril in elderly hypertensive patients with type 2 diabetes. Am J Hypertens 2007; 20(1):90-7.

Zhanying Liu' ${ }^{1}$, Lin Zhang ${ }^{2}$, Ruijie Zhai ${ }^{1}$ and jiarong Lan $^{3}$

${ }^{1}$ Dialysis Room, Changyi City People's Hospital, China ${ }^{2}$ Department of Kidney Medicine, Changyi City People's Hospital, China

${ }^{3}$ Department of Nephrology, Huzhou Hospital of Traditional Chinese Medicine Affiliated Zhejiang University of Traditional Chinese Medicine, China

Correspondence to: Jiarong Lan, Department of Nephrology, Huzhou Hospital of Traditional Chinese Medicine Affiliated Zhejiang University of Traditional Chinese Medicine, 313000, China

E-mail: jiajiec8739@163.com

Received: October 18, 2019; Revised: November 19, 2019; Accepted: December 23, 2019

DOI: https://doi.org/10.29271/jcpsp.2020.09.1000 